\title{
Mit Fischöl den Muskelabbau bei Krebspatienten stoppen
}

Patienten, die sich einer Chemotherapie unterziehen müssen, verlieren oft an Gewicht und Muskelmasse. Wie eine aktuelle Studie in Cancer online jetzt zeigt, könnte eine Nahrungsergänzung mit Fischöl dies verhindern. Zwei Patientengruppen mit neu diagnostiziertem nicht kleinzelligem Lungenkarzinom unterzogen sich der Standardtherapie. Die Probanden einer Gruppe erhielten über zehn Wochen zusätzlich Fischöl in Form von 2,2 Gramm Eicosapentaensäure pro Tag. Bei allen Patienten wurden Gewicht, Muskelmasse und Fettanteil kontrolliert. Die meisten Patienten mit Standardbehandlung verloren durchschnittlich $2,3 \mathrm{~kg}$ während der Chemotherapie. Nur 29\% der Teilnehmer dieser Kontrollgruppe nahmen nicht ab. Dagegen konnten 69 \% der Patienten, die zusätzlich Fischöl erhielten, ihr Körpergewicht konstant halten oder gewannen sogar Muskelmasse hinzu. Zudem zeigte sich ein dosisabhängiger Effekt: Je höher die Eikosapenta-
ensäure-Konzentration im Blut war, desto mehr Muskeln bauten die Patienten auf. Hinsichtlich der Gesamtmasse an Fettgewebe gab es zwischen den Gruppen dagegen keinen Unterschied.

Nach Ansicht der Autoren könnten Patienten während einer Standardkrebstherapie von 2,2 g Fischöl täglich profitieren. Sie hoffen, damit eine Möglichkeit gefunden zu haben, die krebsbedingte Mangelernährung in den Griff zu bekommen. Denn die Supplementation eignet sich auch für Patienten mit anderen Krebsarten oder chronischen Krankheiten, die mit Mangelernährung einhergehen.

\section{Christine Starostzik}

Rachel A Murphy et al. Nutritional intervention with fish oil provides a benefit over standard of care for weight and skeletal muscle mass in patients with nonsmall cell lung cancer receiving chemotherapy. Cancer. Article first published online: 28 FEB 2011. DOI:10.1002/cncr.25709.

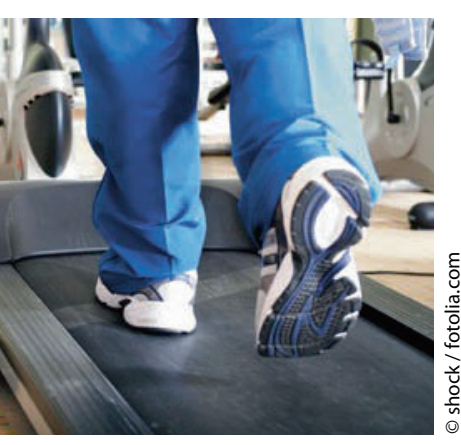

Patienten, die sich einer allogenen Stammzelltransplantation unterziehen, profitieren von regelmäßiger körperlicher Aktivität.

\section{Aktiv gegen Fatigue Sport hilft gegen Erschöpfung bei Stammzelltransplantation}

Absolvieren Patienten ein moderates Trainingsprogramm, ertragen sie die physischen und psychischen Folgen einer allogenen Stammzelltransplantation besser. $\mathrm{Zu}$ diesem Ergebnis kamen Joachim Wiskemann vom Zentralinstitut für Seelische Gesundheit in Mannheim und Kollegen. Sie behandelten randomisiert Patienten, denen eine allogene Stammzelltransplantation bevorstand, in eine Sport- und eine Kontrollgruppe. Die Patienten in der Sportgruppe $(n=40)$ trainierten ein bis vier Wochen bevor sie ins Krankenhaus kamen, während der Therapie und sechs bis acht Wochen nach der Entlassung. Nach einer praktischen Einführung durch einen Spezialisten am Beginn des Programms trainierten die Patienten regelmäßig: zweimal wöchentlich absolvierten sie ein Krafttraining, dreimal ein Ausdauertraining (im Krankenhaus bis zu fünfmal). Zu Hause trainierten sie allein, im Krankenhaus wurden die Patienten zweimal pro Woche bei ihren sportlichen Übungen überwacht. Die Kontrollgruppe $(n=40)$ wies man nur darauf hin, dass sich moderate physische Aktivität während der Therapie positiv auswirkt, sie bekam jedoch keine genaueren Instruktionen. Während des Klinikaufenthaltes hatten alle Patienten $\mathrm{Zu}$ gang zu Trainingsgeräten. Fatigue, Fitness und Distress der Studienteilnehmer wurden zu Anfang der Studie, bei der Einweisung ins Krankenhaus, bei der Entlassung und sechs bis acht Wochen danach standardisiert gemessen. Dabei erfolgte die Ermittlung der Fatigue-Scores über mehrere Fragebögen. In der Trainingsgruppe verbesserten sich die Fatigue-Scores um bis zu $15 \%$, während sie sich in der Kontrollgruppe um bis zu $28 \%$ verschlechterten $(\mathrm{p}<0,01-0.03)$. Auch der Distress $(\mathrm{p}=0,03)$ und die physische Fitness verbesserten sich in der Sportgruppe signifikant (gemessen u.a. an der Anzahl der Meter, die innerhalb von sechs Minuten auf dem Laufband zurückgelegt wurden; $p=0,02$ und der Kraft in den unteren Extremitäten; $p=0,03$ ). Zusammen mit der Fitness verbesserten sich auch die Fatigue-Scores und der Distress.

Dieses teilweise überwachte Trainingsprogramm sei vorteilhaft für Patienten, die sich einer allogenen Stammzelltransplantation unterzogen, folgern die Autoren. Wegen den geringen personellen Anforderungen halten Wiskemann und Kollegen es für sinnvoll, ein solches Programm in die Standardbehandlung zu integrieren.

\section{Carina Jung}

Wiskemann J et al. Effects of a partly self-administered exercise program before, during, and after allogeneic stem cell transplantation. Blood. 2011;117(9):2604-13. 